\title{
Arquitectura del dosel en yuca (Manihot esculenta Crantz) en el bosque seco tropical en Colombia
}

\author{
Canopy architecture in cassava (Manihot esculenta Crantz) in tropical dry \\ forest in Colombia
}

\author{
Salcedo M Jairo ${ }^{1 *}$ Ph.D, Chavarria A Bernardo Ing. Agrónomo, Pérez B Jhonys ${ }^{1}$ M.Sc.
}

Universidad de Sucre, Grupo de investigación PADES (Procesos Agroindustriales y Desarrollo Sostenible), Colombia.

Keywords:

Genotypes; Insertion angle; variety; branching.
Palabras Clave:

Ángulo de inserción; ramificación; altura de planta.
INFORMACIÓN

Recibido: 29-04-2017;

Aceptado: 05-09-2017.

Correspondencia autor:

jairo.salcedo@unisucre.edu.co

\section{Abstract}

The objective of the research was evaluated the influence of soil availability on the architecture of cassava canopy. The work was carried out in the tropical dry forest area of the state of Sucre, Colombia. Experimental plots were established in October 2015, records of plant height, number of branches, foliar insertion angle and branching at twelve months of age, low experimental design in randomized complete blocks, with factorial arrangement. As fixed factors the cassava variety (MCOL 2066, CM 4919-1, SM 1411 and SM 2775) and irrigation (complementary irrigation and without complementary irrigation) were produced. Foliar insertion patterns favored by the absence of irrigation were found, being these of type plagiófilos in the superior third and planificadores in the middle and inferior thirds, what allows greater efficiency in the capture of the radiation. The height of the plant was influenced by the water supply, evidencing the superior growth around $17.9 \%$ under the use of a sheet of 17 millimeters of irrigation during the first months of the crop growth. The irrigation factor promotes in a differentiated way the architectural response of the canopy.

\section{Resumen}

El objetivo de la investigación fue evaluar la influencia de la disponibilidad hídrica del suelo sobre la arquitectura del dosel de yuca. El trabajo se realizó en la zona de bosque seco tropical del departamento de Sucre, Colombia. Las parcelas experimentales se establecieron en octubre de 2015 , registrándose datos de altura de planta, cantidad de ramificaciones, ángulo de inserción foliar y de ramificación a los doce meses de edad, bajo un diseño experimental en bloques completos al azar, con arreglo factorial. Como factores fijos se tuvo la variedad de yuca (MCOL 2066, CM 4919-1, SM 1411 y SM 2775) y el riego (riego complementario y sin riego complementario). Se encontraron patrones de inserción foliar favorecidos por la ausencia de riego, siendo estos de tipo plagiófilos en el tercio superior y planófilos en los tercios medio e inferior, lo que permite mayor eficiencia en la captura de la radiación. La altura de planta fue influenciada por la oferta hídrica, evidenciando superior crecimiento en alrededor de 17,9\% bajo la aplicación de una lámina de 17 $\mathrm{mm}$ de riego durante los primeros meses de crecimiento del cultivo. El factor riego promueve de forma diferenciada la respuesta arquitectónica del dosel. 


\section{Introducción}

La yuca (Manihot esculenta Crantz.) también conocida como mandioca o cassava, se caracteriza por ser una planta de tipo perenne, con tallos formados por la alternancia de nudos y entre nudos. Luego de cierto periodo de crecimiento en este vegetal son emitidas ramificaciones, dependiendo de la variedad. Las hojas de este vegetal están compuestas por una lámina foliar palmeada profundamente lobulada y el peciolo. El número de hojas emitidas por la planta, su longevidad y características fotosintéticas son características específicas de cada variedad e influenciadas por aspectos ambientales de crecimiento (ALVES, 2002; OSPINA y CEBALLOS, 2002).

El producto principal de la planta de yuca, corresponde a raíces de tipo tuberoso, las cuales en recientes años son fuente de alimento básico para una proporción cercana a mil millones de personas a nivel mundial (FAO, 2008). Esto permite considerar dicho producto como cultivo de relevancia en diversos países, principalmente de regiones tropicales (ALVES, 2002).

La arquitectura del dosel (disposición de tallo, ramas y hojas) en esta planta, es un aspecto relevante; que permite conocer el impacto que ejerce la disposición del follaje sobre el proceso intercepción de la radiación incidente; asimismo, su efecto sobre la dinámica en la distribución de fotoasimilados, bajo condiciones de adecuada humedad de suelo tiene un papel crucial en el crecimiento y desarrollo de la planta (FAGERIA et al., 2006; AHUJA et al., 2008). Cabe destacar, que en el estudio de los aspectos estructurales del dosel, la mayor dificultad que afronta el cultivo, corresponde a la gran variabilidad existente entre variedades y dentro de una misma variedad; lo cual obstaculiza su descripción morfológica (OSPINA y CEBALLOS, 2002).

La cantidad de ramificaciones, es un aspecto agronómico importante, que determina la forma arquitectónica de la planta y ejerce influencia sobre el desempeño del cultivo, por otra parte, este rasgo es determinante en la proporción de semillas vegetativas que puede producir la planta (MONTALDO, 1991; CEBALLOS y DE LA CRUZ,
2002). La cantidad de ramificaciones sirve de base para la formación de la cobertura foliar, por tanto, se encuentra relacionado con el índice de área foliar (IAF), que, a su vez, permite profundizar sobre el aspecto productivo en vegetales (LARCHER, 2003; HAY y PORTER, 2006).

La problemática actual que enfrenta el cultivo de yuca en el bosque seco tropical colombiano (bs-T), consiste en el requerimiento de estudios sobre aspectos estructurales del dosel como: número de ramificaciones, ángulo de inserción de hojas por estrato del dosel y su respectiva relación con el índice de área foliar, bajo riego en la oferta ambiental en mención.

En consecuencia el desconocimiento de la arquitectura del dosel de la planta de yuca en el sitio de interés, se tomó como línea de partida para el desarrollo de la investigación en el bs-T Colombiano. Cuyo objetivo consistió en evaluar la influencia de la disponibilidad hídrica sobre la arquitectura del dosel de cuatro genotipos de Manihot esculenta.

\section{Materiales y métodos}

Localización. El presente estudio se realizó al noreste del departamento de Sucre, en el municipio de Corozal, Finca Membrillal, ubicada a una altitud de $103 \mathrm{msnm}$, latitud de $9^{\circ} 09^{\prime} 31^{\prime \prime}$ $\mathrm{N}$ y una longitud de $75^{\circ} 10^{\prime} 8^{\prime \prime}$ oeste, con registro de datos en campo a los doce meses posteriores al establecimiento del cultivo (durante el mes de octubre de 2016). El área de estudio tiene una temperatura promedio anual de $27^{\circ} \mathrm{C}$, humedad relativa promedio de $70 \%$, precipitación promedio anual de $1127 \mathrm{~mm}$, perteneciente a la zona de Bosque Seco Tropical, según HOLDRIDGE (1987).

Suelo. La Tabla 1 muestra la oferta edáfica de nutrientes del suelo perteneciente al ensayo, donde se acentúan las bases del suelo $\mathrm{Ca}, \mathrm{Mg}$ y $\mathrm{Na}$ con valores cuya interpretación es alta; sin embargo cabe resaltar que el efecto de estos elementos sobre las plantas es dependiente de la disponibilidad de asimilación que posean los mismos (SADEGHIAN-KHALAJABADI, 2012). 
Tabla 1. Análisis de propiedades físicas y químicas de suelo, en la Finca Membrillal, Sucre, Colombia. 2015.

\begin{tabular}{|c|c|c|c|c|c|c|c|}
\hline pH M.O. & $\mathbf{P}$ & $\mathrm{Ca}$ & Mg & $\mathbf{K}$ & $\mathrm{Na}$ & Al & $\begin{array}{c}\text { Textura } \\
\text { (Bouyoucos) }\end{array}$ \\
\hline $\begin{array}{ll}(1: 1) \quad(\%) \\
\end{array}$ & Ppm & & & lk & & & \%A $\%$ Ar $\%$ L \\
\hline $6,01 \quad 1,61$ & 7,73 & 9,40 & 3,93 & 0,16 & 2,2 & - & $\begin{array}{lll}59,75 & 23,33 & 21,42\end{array}$ \\
\hline
\end{tabular}

Métodos de análisis: textura: Bouyoucos; $\mathrm{pH}$ : Potenciómetro, relación suelo-agua 1:1, M.O: Walkley-Black modificado, P: Bray II, S y B: Fosfato monocálcico, Al: extracción con $\mathrm{KCl} 1 \mathrm{~N}$, Bases de intercambio: Acetato de amonio, Microelementos: Olsen modificado./ Methods of analysis: texture: Bouyoucos; PH: Potentiometer, soil-water ratio 1: 1, MO: Modified Walkley-Black, P: Bray II, S and B: Monocalcium phosphate, Al: Extraction with $\mathrm{KCl} 1 \mathrm{~N}$, Bases of exchange: Ammonium acetate, Microelements: Modified Olsen; A\%: \%Arena; Ar\%: \%Arcilla; \%L: \%Limo.

Material vegetal. Las variedades de yuca $\mathrm{MCOL}$ 2066 (Chirosa), CM 4919-1 (Veronica), SM 1411(Sinuana) y SM 2775 (Bellotti) correspondieron a los cuatro genotipos objeto de estudio, los cuales se establecieron en campo a mediados del mes de octubre de 2015, con una densidad de población de 10,000 plantas/ha, en unidades experimentales o parcelas con dimensiones de $15 \times 20 \mathrm{~m}$. El montaje del ensayo siguió un diseño factorial bajo un esquema de aleatorización de bloques completos al azar, con cuatro repeticiones, donde se manipularon los factores, riego y variedad de yuca. En este sentido, se tuvo un sector bajo riego, el cual, se constituyó de cuatro parcelas experimentales (una por variedad) y otro sector donde no fue aplicado riego (aporte hídrico de precipitaciones), con igual número de variedades. El manejo agronómico de la plantación fue acorde a las labores de siembra, control fitosanitario y de arvenses; que normalmente se efectúan en la zona (ÁLVAREZ et al., 2002).

Riego. El efecto de riego se analizó a través de un sistema presurizado con cinta de goteo (diámetro de $16 \mathrm{~mm}$, con goteros cada $20 \mathrm{~cm}$ ), distribuidas en campo acorde al arreglo de siembra en hileras, con base a la distribución de plantas en campo ( $1 \mathrm{~m}$ entre plantas e hileras), aportando una lámina de agua correspondiente a $17 \mathrm{~mm}$, durante los primeros cinco meses del ciclo del cultivo, distribuidos de la siguiente forma: primera aplicación el día 15 de octubre de 2015 coincidiendo con el día de siembra de los materiales, posteriormente las siguientes aplicaciones se efectuaron con una frecuencia de tres días, suspendiendo ello cuando las precipitaciones superaron la lámina a aplicar hasta los 150 días de establecido el vegetal.
Variables a medición. Para evaluar la influencia del riego en la estructura del dosel de cada uno de los cuatro genotipos seleccionados, se realizaron mediciones a los doce meses de edad, sobre cuatro plantas por variedad tanto en riego como sin riego, se registró:

\section{Cantidad de ramificaciones por planta, determinadas por conteo.}

Ángulo de ramificaciones (AR) respecto a la horizontal, registrada con transportador, ubicado en una horizontal (definida mediante nivel de burbuja), con cierto grado de perpendicularidad al tallo de la planta, similar a lo realizado por Castaño et al. (2015).

\section{Altura de planta desde la base de la misma (nivel del suelo) hasta la cúspide del dosel.}

Posteriormente, el dosel se dividió en tres estratos de igual altura (superior, medio e inferior), donde se midió el ángulo de inserción (determinado tal como se realizó en el AR) del peciolo de cuatro hojas por estrato del dosel en el tallo.

Las características del hábitat lumínico y la respuesta arquitectónica del vegetal se determinaron mediante el uso del equipo SunScan Canopy Analysis System, el cual registra el índice de área foliar (IAF), así como la radiación fotosintéticamente activa (RFA), tanto en el ápice (lo) como en cualquier estrato del dosel (Iz); variables que, conforme lo expresa Larcher (2003), permiten calcular el coeficiente de extinción de la luz (k), mediante la ecuación de Lambert Beer, modificada por MONSI y SAEKI (Iz= lo. e-k.IAF), para cada tratamiento.

Se adelantaron mediciones debajo de la fronda cuando el sol se encontraba en el zenit, lo que ocurrió entre 11:00 y 14:30 horas, así: en las direcciones norte-sur y este-oeste, así como entre los surcos y dentro de los surcos, para un total de cuatro mediciones por planta.

Análisis de datos. El análisis de los datos consideró un diseño factorial bajo un esquema de aleatorización de bloques completos al azar en el entorno estadístico SAS ${ }^{\circledR} 9.3$ (SAS, 2011) Teniendo como factores fijos: variedad con 
cuatro niveles (MCOL 2066, CM 4919-1, SM 1411 y SM 2775) y riego con dos niveles (riego complementario y sin riego complementario). La comparación de medias se efectuó mediante la prueba de Tukey, con un nivel de significancia del $5 \%$.

\section{Resultados}

\section{Disposición foliar del dosel}

Tercio superior, medio e inferior del dosel. La disposición foliar en el dosel exhibió comportamientos diferentes en los tercios estudiados, dichas variaciones son evidentes en la significancia de los factores que intervinieron en el ensayo. Siendo notorio el efecto positivo que surtió la ausencia de riego, donde se evidenció un arreglo estructural (arquitectónico) del dosel con mayor potencialidad para la interceptación de radiación incidente.

En el tercio superior no se encontró efecto significativo $(P>0,05)$ de los factores riego y variedad. Este exhibió un ángulo de inserción cercano a los $39^{\circ}$ (arreglo plagiófilo: $30^{\circ}-60^{\circ}$ ), sin embargo, a nivel muestral hubo mayor ángulo de inserción con respecto a la horizontal en las parcelas bajo riego. Por otra parte, en el tercio medio del dosel, la disposición foliar fue de tipo planófila (arreglo con ángulos de $0^{\circ}-30^{\circ}$ ) y presentó un efecto significativo $(P<0,05)$ en la interacción de factores riego y variedad, con una media en ángulo de inserción próxima a los $18^{\circ}$. Por último, para el caso del tercio inferior del dosel, se encontró efecto significativo $(P<0,05)$ de los factores riego y variedad, con una media en ángulo de inserción próxima a los $16^{\circ}$ y $17^{\circ}$ respectivamente (Tabla 2 ).

La respuesta de los materiales fue variable, al considerar que algunas de ellas no siguieron la tendencia general, como es el caso de los materiales SM 1411 y CM 4919-1 bajo riego complementario, los cuales exhibieron un aumento significativo en el ángulo de inserción foliar del tercio medio e inferior con respecto al presentado por las mismas variedades en ausencia de riego.
Tabla 2. Media de ángulos de inserción foliar correspondiente al tercio superior, medio e inferior de la planta de yuca, bajo el efecto de dos condiciones hídricas en el bosque seco tropical colombiano para el año 2016.

\begin{tabular}{|c|c|c|c|c|}
\hline \multicolumn{3}{|c|}{$\begin{array}{r}\text { Tercio superior } \\
\left(\mathbf{V}^{n s}, R^{n s}, V_{x} R^{n s}\right)\end{array}$} & \multicolumn{2}{|c|}{ Tercio medio $\left(\mathbf{V} \times \mathbf{R}^{*}\right)$} \\
\hline Variedad & Con riego & Sin riego & Con riego & Sin riego \\
\hline SM 1411 & 49,00 & 38,50 & 31,12 a $A$ & $16,75 \mathrm{~b} \mathrm{~A}$ \\
\hline SM 2775 & 32,10 & 35,50 & 10,37 a B & 10,56 a $A$ \\
\hline MCOL 2066 & 44,70 & 34,60 & 12,81 a $B$ & 17,43 a $A$ \\
\hline CM 4919-1 & 35,00 & 41,80 & 24,25 a A B & 21,81 a A \\
\hline \multicolumn{5}{|c|}{ Tercio inferior $\left(\mathbf{V}^{*}, \mathbf{R}^{*}\right)$} \\
\hline Variedad & Con riego & Sin riego & \multicolumn{2}{|c|}{ Efecto de la variedad } \\
\hline SM 1411 & 24,94 & 17,19 & \multicolumn{2}{|c|}{$21,06 a$} \\
\hline SM 2775 & 11,19 & 11,63 & \multicolumn{2}{|c|}{$11,41 \mathrm{~b}$} \\
\hline MCOL 2066 & 12,56 & 9,31 & \multicolumn{2}{|c|}{$10,94 \mathrm{c}$} \\
\hline CM 4919-1 & 25,94 & 17,44 & \multicolumn{2}{|c|}{26,69 a } \\
\hline $\begin{array}{c}\text { Efecto de } \\
\text { riego }\end{array}$ & $18,65 \mathrm{a}$ & $13,89 \mathrm{~b}$ & & \\
\hline
\end{tabular}

* $y^{\text {ns }}$ ilustran significancia y no significancia. Para el tercio medio, las letras minúsculas, muestran comparaciones dentro de la variedad y letra mayúsculas en columna, indica comparaciones entre cada una de las variedades, igual letra en ambos casos expresas falta de diferencia significativa para el tercio inferior, las letras minúsculas expresas comparaciones entre efectos principales variedad y riego.

Ángulo de ramificación. Los ángulos de las ramificaciones evaluadas en el ensayo no presentaron efecto significativo $(P>0,05)$ de los factores riego y variedad, ni en su interacción, a pesar de esto, a nivel muestral se encontró con una media en ángulo próxima los $40^{\circ}$ (Tabla 3 ), lo cual indicaría ser un rasgo genético poco alterado por el riego y estable entre los diferentes genotipos.

Tabla 3. Media de ángulos de ramificación en yuca bajo el efecto de dos condiciones hídricas en el bosque seco tropical colombiano para el año 2016.

\begin{tabular}{ccc}
\hline Variedad & Con riego & Sin riego \\
\hline SM 1411 & 39,50 & 41,25 \\
SM 2775 & 41,00 & 37,13 \\
MCOL 2066 & 41,33 & 44,00 \\
CM 4919-1 & 35,75 & 41,00 \\
\hline
\end{tabular}

Cantidad de ramificaciones (CR)

De forma general, se presentaron tres ramificaciones por planta para el tratamiento con riego y dos ramificaciones para las plantas sin el efecto de riego, bajo las condiciones del bosque seco tropical colombiano. Dicho comportamiento 
mostró una respuesta positiva a la interacción de la variedad con la aplicación de $17 \mathrm{~mm}$ de agua durante los cinco primeros meses del cultivo.

En la cantidad de ramificaciones en la planta de yuca, se presentó un efecto significativo ( $P$ $<0,05)$ de la interacción de los factores riego y variedad. Se encontró que las variedades SM 1411, MCOL 2066 y CM 4919-1 bajo el efecto de riego, emitieron tres ramificaciones por planta; a diferencia, del material SM 2775 que bajo la misma condición hídrica, presentó dos.

\section{Diámetro, superficie del dosel e interceptación de radiación}

Diámetro del dosel. A partir del análisis de varianza, se obtuvo que esta variable expresó un efecto significativo $(P<0,05)$ del factor variedad. Se evidenció que a pesar de que el diámetro del dosel mostró un comportamiento similar entre las variedades SM 2775, MCOL 2066 y CM 4919-1, se estableció una diferencia del $36,5 \%$ entre las plantas de SM 1411 y SM 2775 a favor de este último (Figura 1).

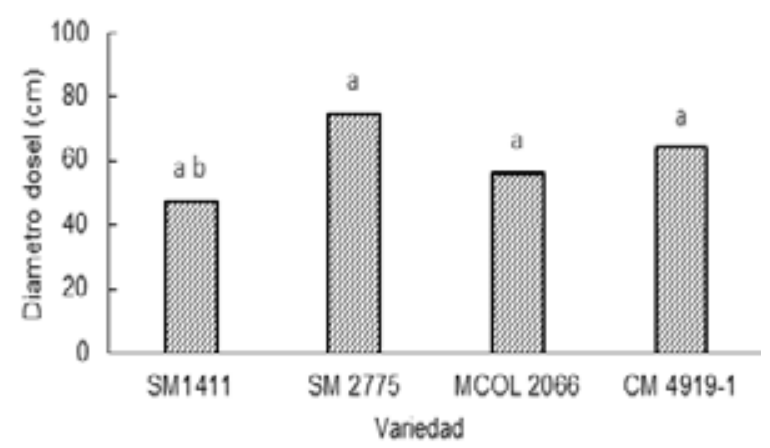

Figura 1. Media de diámetro del dosel en cuatro variedades de yuca bajo el efecto de dos condiciones hídricas en el bosque seco tropical colombiano para el año 2016.

Las letras minúsculas que acompañan los promedios correspondientes al efecto de las variedades, permiten relacionar la significancia que se presentaron los datos en dicho factor.

Superficie del dosel e Interceptación de radiación (índice de área foliar y coeficiente de extinción de luz). El índice de área foliar (IAF) en yuca presentó un efecto significativo $(P<0.05)$ del factor riego, encontrándose el mayor IAF en SM
2775 , con un área foliar mayor en alrededor del $70 \%$, con respecto al área de suelo destinada para la planta, bajo la ausencia de riego, la cual, no expuso diferencias significativas con la variedad CM 4919-1 bajo riego, con una media entre ambas correspondiente a 0,99 .

Las medias generales con riego y sin riego ofrecieron datos de 1,0 y 1,4 de forma respectiva, lo que representó claramente la relación inversa con el manejo hídrico; cuya relación da como resultado concluyente; incrementos en el índice de área foliar a los doce meses posteriores a siembra en las variedades de yuca estudiadas, al encontrarse expuestas a una disminución en la oferta hídrica durante los primeros cinco meses de cultivo.

El coeficiente de extinción de luz encontrado en yuca, mostró ausencia del efecto significativo $(P>$ 0,05 ) de los factores riego, variedad e interacción, con una media cercana a los 0,47 con riego y 0,54 sin riego (adimensional), sin embargo, dichos datos a nivel muestral presentaron mayor coeficiente de extinción de luz en plantas que llevaron a cabo su crecimiento y desarrollo en condiciones sin riego durante los meses iniciales del cultivo.

Altura de planta. La altura de planta mostro efecto significativo $(P<0,05)$ de los factores riego y variedad, encontrándose respuesta positiva a la adición de riego, reflejado en incrementos de altura de planta de $17,9 \%, 13,2 \%$ y $4,3 \%$, para MCOL 2066, SM1411 y CM 4919-1 respectivamente y respecto a sus equivalentes en ausencia de riego (Tabla 4). Para el caso del material SM 2775, este presentó incrementos del $30 \%$ de altura, al encontrarse sin riego durante los primeros meses de cultivo.

Tabla 4. Altura de la planta de yuca $(\mathrm{cm})$ bajo el efecto de dos condiciones hídricas en el bosque seco tropical colombiano para el año 2016.

\begin{tabular}{ccc}
\hline Variedad & Con riego & Sin riego \\
\hline SM 1411 & 274,75 a A & 238,50 a B C \\
SM 2775 & 216,25 b A & 305,75 a A B \\
MCOL 2066 & 270,75 a A & 222,25 a B C \\
CM 4919-1 & 268,50 a A & 257,00 a B C \\
\hline
\end{tabular}

Las letras minúsculas que acompañan las medias, de forma horizontal permiten relacionar la significancia que se presentó en el factor riego y las letras mayúsculas de forma vertical, la significancia entre variedades. 


\section{DISCUSIÓN}

Disposición foliar. De forma general, el ángulo en el tercio superior, mostró una distribución foliar plagiófila, la cual permite el aprovechamiento de una fracción de la radiación proporcionada por el oferta ambiental característica de la zona, adicionalmente ello favorece, el paso de la radiación no interceptada hacia los tercios inferiores del dosel, apropiada distribución de la radiación insiérete en el dosel y disminución de la probabilidad de fotoinhibición (LAMBERS et al., 2008). Lo anterior coincide con lo encontrado en la planta de maíz por MONTEMAYOR et al. (2006), quienes resaltan, que al establecerse un patrón de hojas verticales en la parte superior del dosel, seguido de una tendencia más horizontal en los estratos inferiores, se genera una menor interceptación de la radiación en las hojas superiores permitiendo el paso de una mayor proporción de luz hacia las hojas inferiores. Dando como resultado una distribución más equitativa de la radiación sobre el total del área foliar.

La distribución foliar del tercio medio presentó un menor ángulo de inserción con respecto al tercio superior. Lo cual, facilitó la presencia de una mayor área foliar expuesta a la radiación solar, que a su vez, permitió interceptar la radiación no captada por el estrato superior. Dicha distribución exhibió variaciones bajo el efecto de los factores de estudio mostrando una diferencia del $53,8 \%$ a favor en la variedad industrial SM 1411, expuesta a una lámina de $17 \mathrm{~mm}$ empleada como tratamiento. Echo que repercute de forma positiva en la obtención de energía disponible para los procesos fisiológicos requeridos por las plantas (SQUIRE, 1990; KJAERSGAARD et al., 2007).

Los materiales de yuca evaluados en el tercio inferior, de forma general, ostentaron una distribución planófila, sin embargo, las medias de las variedades SM 2775 y MCOL 2066 muestran una tendencia de distribución más horizontal, representada por una disminución en el ángulo de inserción de las hojas del 52,6\% y $50,4 \%$ respectivamente; estas características son propias de los materiales que se relacionan con un potencial mucho más competitivo en captación de luz, siendo determinante en la tasa de fotosintética que pueda experimentar el vegetal y por consiguiente en el abastecimiento de asimilados en la hoja (ENCISO y GÓMEZ, 2004). $\mathrm{Al}$ analizar el efecto principal del riego, se obtuvo que los materiales de yuca experimentaron una tendencia con mayor horizontalidad en ausencia de riego complementario, ello sugiere que la estructura del dosel puede modificarse como mecanismo de respuesta a estímulos externos, percibidos por las plantas durante su desarrollo. Lo anterior coincide con lo encontrado por varios autores (ALVES, 2002; OSPINA y CEBALLOS, 2002), quienes resaltan una alta variabilidad de características morfológicas y fisiológicas entre las diferentes variedades e incluso entre plantas de la misma variedad al ser influenciadas por aspectos ambientales de crecimiento.

Ángulo de ramificación. Para el caso de las plantas de yuca evaluadas, la expresión de muchas de las características fenotípicas, incluida el ángulo de ramificación, alcanzan a experimentar cierto grado de variabilidad, en algunas ocasiones más marcada que en otros, y dichos cambios se encuentran estrechamente influenciados por la oferta ambiental, al igual que por las características edáficas del terreno. Como consecuencia, la arquitectura típica de una determinada variedad o conjunto de variedades situadas en un ambiente específico, pueden presentar un comportamiento diferente al establecerse en otro sitio, lo que sería un tema de interés por evaluar, donde seguramente se podrían encontrar diferencias significativas en los materiales, soportándose en lo encontrado por CEBALLOS y CRUZ (2002).

Cantidad de ramificaciones (CR). EI comportamiento que siguió esta variable respuesta, muestra cierta dependencia de la variedad, como lo reflejó el material SM 2775 , el cual no siguió la tendencia general de mayor número de ramificaciones bajo riego. Coincidiendo con lo encontrado en otro estudio donde se reportó que el número de ramificaciones es propio de cada variedad, basado en los estudios realizados sobre las variedades MCOL 113 y MCOL 22 donde normalmente la primera variedad en cuestión, produce 4 ramas y ocasionalmente 3 en cada punto de ramificación, mientras que la variedad MCOL 22 produce 3 en la mayoría de los casos y algunas veces 2 (MONTALDO, 1991). De igual 
forma, esta tendencia puede ser explicada por medio de los hallazgos de otros investigadores (SÁNCHEZ y PARODI, 2001; ACOSTA et al., 2006) quienes se refieren a los niveles de ramificación, hábito de ramificación y al tipo de planta como características con alta penetrancia y expresividad específica.

La cantidad de ramificaciones se encuentra fuertemente influenciada por las características físico-químicas del suelo, las cuales son tan cambiantes que pueden establecerse diferencias e incluso dentro de un mismo predio, pudiendo generar alteraciones en el desarrollo de las plantas (LOBO, 2006). Al encontrar que las medias generales de ramificación se hallaron influenciadas por la interacción de los factores variedad y riego. Tomando como base los reportes efectuados por los autores citados en párrafos anteriores, se puede concluir que la información experimental obtenida en el ensayo con respecto a número de ramificaciones, es producto de la respuesta de la variedad a la oferta hídrica, que a su vez pudo permitir una mayor disponibilidad de nutrientes en estado de solución (líquido) para las plantas de yuca durante los primeros meses de establecimiento.

\section{Diámetro, superficie del dosel e interceptación de radiación.}

Diámetro. Al comparar la media del material SM 1411 con las variedades SM 2775 y CM 4919-1; se encontró que esta característica difirió. Dicha variación se produjo a causa de la oferta edáfica y disponibilidad de nutrientes en el área de estudio, al igual que lo ocurrido en el ítem anterior con respecto al número de ramificaciones que manifestaron las plantas evaluadas. Afirmación soportada por estudios que reportaron reducciones en el dosel de la planta de yuca en materiales procedentes de semillas obtenidas de terrenos en ausencia de fertilización (MOLINA y EI-SHARKAWY, 1995).

Superficie del dosel. Las plantas en ausencia de riego experimentaron una mayor área total de la superficie foliar por área de suelo proyectada, a diferencia de las plantas bajo el efecto de riego. Comportamiento manifestado a causa del estímulo ejercido por la poca disponibilidad de agua sobre el mecanismo de respuesta fisiológica propio que exhibe la planta de yuca. Adicionalmente, se han encontrado resultados similares, donde inicialmente, la planta de yuca se auto regulo, efectuando un uso más eficiente del recurso hídrico, continuando con una reducción en el crecimiento de los diferentes órganos de la planta, que se traduce en una disminución en la acumulación de biomasa; la cual se interrumpe al reestablecerse las condiciones hídricas necesarias para el óptimo desarrollo del cultivo (EL SHARKAWY, 1993; CAYÓN et al., 1997). Exhibiendo en esta etapa post-stress una tendencia positiva sobre la formación de hojas nuevas con tasas de fotosíntesis más altas, que permiten una recuperación rápida de los materiales afectados, expresado en un incremento del índice de área foliar coincidiendo con lo reportado por CHEN et al. (1997).

Interceptación de radiación. La tendencia planófila en inserción foliar, se puede apreciar al relacionar la disposición de las hojas en variedades sin efecto de riego para los tercios: medio e inferior; los cuales experimentaron una tendencia más horizontal expresada con porcentajes de 6,5, 15,3 y 25,5 respectivamente. Lo anterior permite una mayor absorción de luz que se traduce en incrementos sobre la eficiencia en captación de radiación fotosintéticamente activa, necesaria para el proceso fotosintético, esto coincide con lo encontrado por CASTILLO y SENTIS (2001). Adicionalmente, dicho coeficiente de extinción de igual forma, se relaciona con el índice de área foliar exhibiendo un dato más elevado en tratamientos que ofrecen un mayor IAF comportamiento similar fue reportado en diferentes cultivos (CAMACHO et al., 1995; MÁRQUEZ et al., 2010).

Altura de planta. Al evaluar el efecto del factor variedad, se obtuvo, que aunque el material SM 2775 presentó una diferencia del $30 \%$ en altitud a favor del tratamiento sin riego, el resto de materiales presentaron un comportamiento similar ante el efecto principal del mismo factor, pero en presencia de riego. Lo cual se explica, al relacionar la altura de planta de yuca como aspecto de crecimiento e indicador de desarrollo relevante, el cual se encuentra directamente influenciado por la interacción genotipo - ambiente. Esto coincide 
con lo encontrado por OSPINA y CEBALLOS (2002). Cada variedad o clon, puede experimentar ciertas variaciones en su morfología y fisiología de acuerdo al estímulo externo que incida sobre ella, como es el caso, de los genotipos objeto de estudio. Ajustándose a lo reportado en girasol por (AGUILAR et al., 2005).

Al evaluar el efecto principal del factor riego, se encontró que la variación existente entre los cuatro materiales establecidos en presencia y ausencia de riego fue significativa, evidenciándose de forma muestral y estadística, que las variedades a excepción de SM 2775, responden de forma positiva a la aplicación de riego incrementando su altura en un $17,9 \%, 13,2 \%$ y $4,3 \%$ de forma respectiva para los materiales MCOL 2066, SM1411 y CM 4919-1. Hallazgo que coincide con lo reportado por otros autores (ARAUJO et al., 2013; PASTRANA et al., 2015).

El material SM 2775 presentó variabilidad bajo el efecto del factor variedad, y fue el que superó a los demás, mostrando un incremento del 16\% en altura con respecto a la variedad CM 4919-1.
Dicho comportamiento igualmente explicado por la alta variabilidad de los genotipos reportada por (OSPINA y CEBALLOS, 2002).

\section{Conclusiones}

Las cuatro variedades de yuca evaluadas, se ajustan a dos patrones de inserción foliar en el dosel, acentuándose ángulos de inserción plagiófila en el tercio superior y planófila en los tercios medio e inferior, estableciendo una disposición del dosel que permite una captura más eficientemente de la radiación.

La aplicación de $17 \mathrm{~mm}$ como lámina de agua durante las etapas iniciales del cultivo influyó de forma positiva sobre la altura de la planta en las variedades de yuca evaluadas.

\section{Agradecimientos}

Los autores dan gratitud al sistema general de regalías Colciencias, quien permitió la financiación del presente trabajo, desarrolla en el proyecto: Desarrollo agroindustrial delos cultivos de yuca y ñame en el departamento de Sucre.

\section{Referencias}

ACOSTA, R. A.; Tamayo, A. C.; PALACIOS, R. (2005). Caracterización morfológica y extracción de adn de 11 clones de yuca (manihot esculenta crantz.) en la universidad earth, Costa Rica.

AGUILAR, L.; ESCALANTE, J.; FUCIKOVSKY, L.; TIJERINA, L. y MARK, E. 2005. Área foliar, tasa de asimilación neta, rendimiento y densidad de población en girasol. Terra Latinoamericana, 23(3), 303 - 310.

AHUJA, L.; REDDY, V.R. ; SASEENDRAN, S. A.; and QUIANG, Y. 2008. Response of crops to limited water. Understanding and modeling water stress effects on plant growth processes $1^{\text {st }}$ ed. American Society of Agronomy. Madison, WI, USA.

ÁLVAREZ, E.; BELLOTTI, A.; ARIAS, B.; CADAVID, L.F. y Llano, G. 2002. Guía práctica para el manejo de las enfermedades, las plagas y las deficiencias nutricionales de la yuca. $1^{\text {ra }}$ ed. Centro Internacional de Agricultura Tropical. Cali, COL.

ALVES, A. 2002. Cassava botany and physiology. In: R.J. Hillocks et al., editors, Cassava: Biology, Production and Utilization. $1^{\text {st }}$ ed. CABI Publishing, GBR. p. 67-89.

ARAÚJO, J.; PAEZ, R.; AMORIM, P.; COMASSETTO, F. y SILVA, S. 2013. Características morfológicas e produtivas da maniçoba cultivada sob lâminas hídricas e doses de nitrogênio. Revista Brasileira de Saúde e Produção Animal, 14(4): 609-623. 
CAMACHO, R.; GARRIDO, O. y LIMA, M. 1995. Caracterización de nueve genotipos de maíz (Zea mays L.) en relación a area foliar y coeficiente de extinción de luz. Scientia Agricola, Piracicaba, 52(2): 294-298.

CASTAÑO, A.; CHICA, H.; OBANDO, D. y RIAÑO, N. 2015. Arquitectura del dosel e interceptación de la radiación solar por tres especies forestales nativas en Colombia. Cenicafé 64(1): 19-30.

CASTILLO, F. y SENTÍS, F.C. 2001. Agrometeorología: Mundi-Prensa libros, Madrid, ESP.

CAYÓN, M.; EL-SHARKAWY M. and CADAVID, L. 1998. Leaf gas exchange of cassava as affected by quality of planting material and water stress. Photosynthetica 34: 409-418.

CEBALLOS, H. y DE LA CRUZ, G. 2002. Taxonomía y Morfología de la Yuca. En: B. Ospina, y H. Ceballos, editores, La yuca en el tercer milenio: sistemas modernos de producción, procesamiento, utilización y comercialización. $1^{\text {ra }}$ ed. CIAT, COL. p. 17-34.

CHEN, J.M.; PLUMMER, P.S.; RICH, M.; GOWER, S.T. and NORMAN, J.M. 1997. Leaf area index measurements. Agricultural and Forest Meteorology, 140(1): 257-268.

ENCISO, B. y GÓMEZ, C. 2004. Comparación de las respuestas de cuatro cultivares de mora (Rubus sp.) a las variaciones del factor luz. Agronomía Colombiana, 22(1): 46-52.

EL-SHARKAWY, M. 1993. Drought-Tolerant Cassava for Africa, Asia, and Latin America. BioScience (43): 441-451.

FAGERIA, N.; BALIGAR, V. and CLARK, R. 2006. Physiology of crop production. $1^{\text {st }}$ ed. Food Products Press, N.Y., USA.

FAO. 2008. Yuca para la seguridad alimentaria y energética. FAO. http://www.fao.org/Newsroom/es/ news/2008/1000899/index.html (consultado 31 oct. 2016)

GIL, J., y BUITRAGO, J. 2002. La Yuca en la alimentacion animal. En: B. Ospina, y H. Ceballos, editores, La yuca en el tercer milenio: sistemas modernos de producción, procesamiento, utilización y comercialización. $1^{\text {ra }}$ ed. CIAT, COL. p. 527-569.

HAY, R. y PORTER, J. 2006. The physiology of crop yield. $1^{\text {st }}$ ed. Blackwell Pub., Oxford, GBR.

HOLDRIDGE, L. 1987. Ecología basada en zonas de vida. $3^{\text {ra }}$ ed. Instituto Interamericano de cooperación para la agricultura, San José, CRC.

KJAERSGAARD, J.; PLAUBORG, F. and HANSEN, S. 2007. Comparison of models for calculating daytime long-wave irradiance using long term data set. Agricultural and forest Meteorology, 143(1): 49-63.

LARCHER, W. 2003. Physiological plant ecology. $1^{\text {st }}$ ed. Springer, Berlin, GBR.

LOBO, R. 2006. Caracterización de yuca (Manihot esculenta Crantz). En: R. JIMÉNEZ et al., editores, Conservación in situ de cultivos nativos y parientes silvestres. Comunicación Técnica. INIEA (Instituto Nacional de Investigación y Extensión Agrícola), PER. p. 106-118.

LÓPEZ, M. y LÓPEZ, R. 1984. Raíces y Tubérculos. Pueblo y educación. La Habana, CUB.

LAMBERS, H.; CHAPIN III.; Stuart, F.; PONS, T. L. 2008. Plant physiological ecology. Springer, New York. 
MARQUEZ, J.; ANDRESSEN, R. y DENNET, M. 2010. Intercepción de radiación solar por el cultivo de cebolla (Allium cepa) en condiciones tropicales semiáridas. Revista Unellez de Ciencia y Tecnología (28): 43-46.

MONTALDO, A. 1991. La yuca o mandioca. $2^{\text {ra }}$ ed. IICA (Instituto Interamericano de Ciencias Agrícolas), San José, CRC.

MONTEMAYOR, J.; ZERMEÑO, A.; OLAGUE, J.; ALDACO, R.; FORTIS, M.; SALAZAR, E.; RODRÍGUEZ, J. y VÁZQUEZ C. 2006. Efecto de la densidad y estructura del dosel de maíz en la penetración de la radiación solar. Phyton (B. Aires) 75: 47-53.

MOLINA, J.L. and EL-SHARKAWY M.A. 1995. IncreasIng crop produc tivity in cassava by fertilizing production ol planting material. FieldCrops Aes (44): 151-157.

OSPINA, B. y CEBALLOS, H. 2002. La yuca en el tercer milenio. $1^{\text {ra }}$ ed. CIAT, Cali, COL.

PASTRANA, F.; ALVIZ, H. y SALCEDO, J. 2015. Respuesta de dos cultivares de yuca a la aplicación de riego en condiciones hídricas diferentes. Acta Agronómica, 64(1): 48-53.

SADEGHIAN-KHALAJABADI, S. 2012. Efecto de los cambios en las relaciones de calcio, magnesio y potasio intercambiables en suelos de la zona cafetera colombiana sobre la nutrición de café (Coffea arabica L.) en la etapa de almácigo. Tesis dr. Universidad Nacional de Colombia, Medellín, COL.

SÁNCHEZ, H.I. y PARODI, J.L. 2001. Diversidad de yuca (Manihot esculenta Crantz) en Jenaro Herrera, Loreto-Perú. Boletín técnico $n^{\circ}$ 28. Instituto de Investigación de la Amazonía Peruana, PER.

SAS Institute. 2011. User's guide: Statistics. SAS Inst., Cary, NC, USA.

SQUIRE, G. 1990. The physiology of tropical crop production. $1^{\text {st }}$ ed. C.A.B. International for the Overseas Development Administration, Wallingford, Oxon, GBR. 